\title{
El desarrollo de la Clasificación Decimal Universal: 1992-2008 y más allá
}

\author{
Aida Slavic*, Maria Inês Cordeiro*, Gerhard Riesthuis*
}

Resumen: El artículo pone en relieve algunos aspectos de la política de gestión de la CDU desde 2007 en adelante. Tras una revisión de la larga historia de modernización de esta clasificación, que comenzó en la década de los 60, continuando el proceso de revisión y desarrollo del esquema en los 1990, se resumen los cambios y políticas más importantes de la historia reciente de la CDU. Finalmente, se presentan los puntos de vista del nuevo equipo editorial del CDU establecido en 2007, y su nueva política que se enfoca en la organización y eficiencia del trabajo editorial y la mejora de los productos de la CDU.

Palabras clave: CDU, Clasificación Decimal Universal, revisión, cambios, desarrollo, gestión.

\section{The development of the Universal Decimal Classification: 1992-2008 and beyond}
Abstract: This paper highlights a number of features of the UDC management policy for 2007 and thereafter. Following an overview of the long bistory of modernization of the classification, which started in the 1960 s, with continued revision and development in the 1990s, the major changes and policies in the recent history of UDC revision are summarized. The perspectives of the new UDC editorial team, which was established in 2007, and its policy regarding the improved organization and efficiency of editorial work and the improvement of UDC products, are discussed.

Keywords: UDC, Universal Decimal Classification, revision, changes, development, management.

Este trabajo es una versión abreviada y revisada de la versión inglesa publicada en el International Cataloguing and Bibliographic Control, en junio de 2008. (traducido por Edgardo Civallero Miembro del Revision Advisory Committee, CDU Correo-e: edgardocivallero@gmail.com.

* Consorcio CDU (UDCC), La Haya. Correo-e: aida.slavic@udcc.org; ines.cordeiro@udcc.org; gerhard.riesthuis@udcc.org.

Recibido: 7-2-07; 2. ${ }^{a}$ versión: 19-12-08. 


\section{Introducción}

Los usuarios de la CDU, o de cualquier otro sistema de clasificación de características similares, a menudo se enfrentan a los resultados finales del proceso evolutivo de un esquema determinado sin conocer las razones o políticas que lo han conducido a esos desarrollos particulares. Desde 1992, la CDU ha sido gestionada por el Consorcio de la CDU (UDCC, Universal Decimal Classification Consortium) y, durante ese período, ha sido intensamente revisada. Hasta 2008 se han elaborado quince ediciones anuales del archivo maestro de referencia (MRF) de la CDU estándar, estimándose que alrededor de un $40 \%$ de la clasificación es diferente, en la actualidad, de su versión de 1990. Una perspectiva general de los cambios introducidos en la clasificación se encuentra publicada en el sitio web del UDCC (http://www.udcc.org/major_changes.htm). Algunos de estos cambios fueron discutidos hace años o incluso décadas, y han sido reportados en informes de investigación, artículos y libros (por ejemplo Freeman y Atherton, 1968; Foskett, 1973; Dahlberg, 1971; etc.).

En 2006-2007 se estableció un nuevo equipo editorial de la CDU; este artículo explica cómo ve dicho equipo su conexión con el pasado y qué prioridades reconoce para el futuro. En primera instancia, revisamos algunas de las circunstancias que condujeron a cambios significativos en los procesos de mantenimiento, distribución y revisión de la CDU durante el período 1993-2006. Considerando el enorme volumen de trabajo que ha tenido lugar en años recientes en relación con la CDU, proveeremos una perspectiva de aquellos cambios que los usuarios pueden esperar en un futuro próximo.

\section{La evolución de la CDU desde la perspectiva de los editores de la clasificación}

\subsection{Período 1960-1990: buscando la mejor forma de mejorar la CDU}

Merece recordarse que en la década de los años sesenta, los profesionales de la información estaban muy desencantados con las clasificaciones biblioteconómicas en general. Esto ocurría con la mayoría de los sistemas más usados, como la Clasificación Decimal Dewey (CDD), la Clasificación de la Biblioteca del Congreso (LC), la Clasificación Bibliográfica Bliss (BC) o la CDU. La estructura de estos sistemas era esencialmente estática, basada principalmente en una perspectiva decimonónica del conocimiento y de la ciencia. En aquella época existía una profunda preocupación sobre los procesos de organización e indización de documentación científica, tareas para las cuales los mencionados sistemas parecían no estar preparados. Florecieron las discusiones sobre cómo tales sistemas (aún usados a nivel mundial) podrían ser mejorados y adaptados a esos propósitos, si es que tal objetivo era posible. 
Como reacción a esta situación, la Federación Internacional de Documentación (FID, International Federation of Documentation), propietaria de la CDU en aquel momento, intensificó la modernización y expansión de la clasificación para actualizarla con una estructura de conocimiento moderna. Hacia comienzos de la década de los ochenta, la edición completa de la clasificación había sobrepasado las 200.000 clases, como resultado del trabajo de numerosos paneles temáticos nacionales e internacionales. La tarea no estaba bien coordina$\mathrm{da}$, y la plétora de conceptos - y la forma incoherente en la que éstos eran agregados al sistema- contribuyeron a crear dificultades en su gestión y mecanización.

Para finales de la década de los ochenta ya se habían generado abundantes propuestas orientadas a superar tales dificultades (Freeman y Atherton, 1969; Lloyd, 1972; Wellisch, 1971; Dahlberg, 1971: 24). Durante las décadas de los sesenta y los setenta muchos expertos en clasificación coincidieron con respecto a varios puntos importantes. Desde una perspectiva de gestión, resultaba claro que la automatización era esencial para el mantenimiento del esquema, y que lo más práctico sería mantener el tamaño del mismo a un nivel de edición "media". En términos de contenido, muchos argumentaron que el esquema debía ser mejorado estructuralmente, y que el análisis de facetas y la eliminación de los términos compuestos representados por notaciones simples sería el camino más apropiado a seguir.

A principios de 1983, la FID comisionó un estudio externo destinado a hallar un mejor camino para gestionar la CDU. Los resultados del estudio fueron publicados en 1984 y reportaron, como resultado positivo, la propuesta de una nueva estructura de gestión de la CDU; dicha propuesta ayudó, más tarde, a manejar el problema de automatizar el proceso de mantenimiento del esquema, iniciando un examen estratégico de las necesidades de revisión y dando los pasos necesarios para solucionar errores en la estructura de la clasificación (Gilchrist, 1992).

El Consejo de gestión de la CDU (UDC Management Board) fue creado en 1986 para implementar los cambios y, en 1989, decidió establecer un Grupo de trabajo para el desarrollo del sistema de la CDU (Task Force for UDC System Development) compuesto por miembros internacionales. En un breve período de tiempo, dicho grupo preparó una estrategia que ofrecía un plan concreto para el desarrollo de la CDU a largo plazo. Se revisaron, resumieron y actualizaron recomendaciones que estaban dispersas en artículos de investigación y en estudios de la década de los sesenta; las mismas fueron incluidas en el informe final del Grupo de trabajo, presentado en febrero de 1990 (FID Internal Documentation, 1990). Este informe fue la base para los procesos de gestión, mantenimiento y revisión que fueron registrados, un año después, en las "Directrices para la creación de una versión estándar de la CDU” (Guidelines for the creation of a standard version of UDC, FID Internal Documentation, 1991). 


\title{
2.2. El veloz ritmo del cambio desde 1990 a 2006
}

El año 1990 marcó un nuevo comienzo para la gestión de la CDU. En 1991, por recomendación del Grupo de trabajo, se elaboraron las «Directrices para la creación de una versión estándar de la CDU». El 1 de enero de 1992, la FID transfirió los derechos de la CDU al recientemente creado Consorcio de la CDU (UDCC, Universal Decimal Classification Consortium), un grupo de editores establecidos como institución sin fines de lucro, con base legal en los Países Bajos y registrada como una fundación neerlandesa. Tras el cambio de propiedad, el proceso de revisión de la CDU y la toma de decisiones se centralizaron, y fueron transferidos a un Comité de revisión, compuesto por dos o tres miembros y un editor en jefe. Este hecho posibilitaría el desarrollo de procesos de revisión más veloces y reduciría los costos de mantenimiento de la clasificación, respondiendo a la primera prioridad del UDCC: modernizar el esquema, reducir sus costos de mantenimiento y distribución a través de la automatización, y lograr que su gestión fuese sustentable.

El primer paso hacia la automatización fue completado en 1993, y consistió en la reducción de tamaño de la que era, en tiempos de la FID, la edición "completa" de la CDU; esta edición fue convertida en un sistema de 60.000 clases, más manejable, que desde entonces fue designado como UDC Master Reference File o MRF (Archivo maestro de referencia de la CDU). Ese mismo año, Gerhard Riesthuis y David Strachan crearon una base de datos en CDS/ISIS para el MRF, basándose en un archivo de texto de 1985 de la edición "media" de la British Standard Institution (BSI), a la cual se agregaron subsiguientes extensiones y correcciones.

El segundo - - y más difícil- paso fue la revisión de todo el contenido de la CDU, llevado a cabo por un editor en jefe junto a un reducido equipo de investigadores. De acuerdo al informe del Grupo de trabajo, "se previó la reestructuración total de la clasificación en forma organizada a lo largo de un período de 10 años". El informe esbozaba un posible plan de acción, y algunas de sus recomendaciones fueron bastante específicas; p.ej.:

\begin{abstract}
"Se sintió que, en la actualidad, un problema general es la confusión entre enumeración de los [términos] compuestos y el facetado, y que el último proceso es el que debería ser apoyado. Se acordó que deberían realizarse todos los esfuerzos necesarios para mantener una notación expresiva siempre que fuese posible. [...] Se necesitaría una revisión cuidadosa de los auxiliares, tanto generales como especiales, dado que, especialmente entre los últimos, algunos conceptos se repiten, lo cual ya ocurre en las tablas principales de clasificación; en esos casos deberían usarse dos puntos. Serían necesarias algunas simplificaciones en las subdivisiones de tiempo, y la tabla de áreas, la cual provee actualmente una mezcla de mundo histórico y real, necesitaría ser corregida". (FID Internal Document, 1990: 7).
\end{abstract}

Asignada al cargo de editora en jefe en 1990, I. C. McIlwaine dirigió, hasta 2006, la desafiante tarea de revisión junto a un equipo de asistentes. El núcleo 
del equipo editorial estaba compuesto por expertos clasificadores: Geoffrey Robinson, en aquel momento editor de la edición BSI de la CDU; Vanda Broughton, editora asociada de la Clasificación Bibliográfica Bliss; y Nancy Williamson, una reconocida investigadora y autora en el campo de la clasificación, quién previamente había trabajado en una conversión de la LCC a formato legible por máquina.

Durante el período entre 1990 y 2008 se lanzaron 15 versiones del MRF de la CDU, y, aunque el plan era mantener el tamaño del esquema inalterado, el MRF aumentó su tamaño de 60.000 a 67.000 registros. Durante dicho período se revisaron cuidadosamente las siguientes clases, las cuales fueron expandidas con nuevos conceptos:

Auxiliares comunes de lugar (en proceso, desde 1994)

Auxiliares comunes de tiempo (2003)

Auxiliares comunes de propiedades (1999)

Auxiliares comunes de relaciones, procesos (2003)

Auxiliares comunes de personas (2001, 2002, 2003)

$\begin{array}{lll}004 & \text { Computación } & (1994) \\ 005 & \text { Gestión } & (2001) \\ 2 & \text { Religión } & (2000,2001,2006) \\ 338.48 & \text { Turismo } & (1999) \\ 364 & \text { Bienestar social } & (2000) \\ 502 / 504 & \text { Ecología } & (1999) \\ 60 & \text { Biotecnología } & (2002) \\ 61 & \text { Medicina } & (\text { en proceso, desde 1996) } \\ 791 & \text { Cine } & (2000) \\ 8 & \text { Lenguas. Literatura } & (1992) \\ 91 & \text { Geografía } & (1994) \\ 93 / 94 & \text { Historia } & (1994)\end{array}$

Además, muchas clases recibieron una "limpieza» rápida de duplicaciones y conceptos obsoletos, p.e. los "Auxiliares comunes de persona", la clase 37 (Educación), la clase 64 (Ciencias domésticas) y la clase 712 (Arquitectura). Se agregaron nuevos conceptos a las clases 51 (Matemáticas), 53 (Física) y 78 (Música), por ejemplo, y se revisaron las tablas auxiliares especiales para la clase 33 (Economía).

De todas las propuestas presentadas por el Grupo de trabajo y adoptadas en las revisiones desde 1993, la que más afectó a los usuarios de la CDU fue la abolición de la llamada "regla de los 10 años", que fue considerada necesaria para facilitar la tarea de revisión (FID Internal Documentation, 1990; McIlwaine, 1990). Esto permitió que los números vacíos de la CDU fueran inmediatamente reutilizados para denotar conceptos diferentes, y, por ende, poner más requerimientos en la apropiada identificación de los números de la CDU durante el proceso de intercambio y actualización de la clasificación. 
Otro aspecto importante del proceso de revisión desarrollado durante ese período fue el facetado de la CDU. Esto requirió la investigación de esquemas facetados ya existentes, especialmente la Clasificación Bibliográfica Bliss, con el objeto de explorar la posible aplicación de la misma estructura a la CDU. Cualquier revisión de esta categoría tomaría más tiempo de lo usual para ser completada, y mostraría algunas repeticiones en el proceso. Así, por ejemplo, la primera propuesta para una tabla de "Religión" totalmente facetada fue lanzada en 1996 por V. Broughton, mientras que la versión final de la nueva clase fue presentada en 1999 y aún se necesitaron siete años más para que se completaran todas las religiones. De manera similar, completar la propuesta de revisión y reestructuración de la clase 61 (Medicina) en forma facetada tomó más de diez años.

El proceso de revisión de la CDU ha sido descrito en numerosos artículos, especialmente por I.C. McIlwaine (1990, 1993, 1995, 1996), N. Williamson (1990, 1994) y V. Broughton (1998). En más de una ocasión, I.C. McIlwaine notó que no siempre era fácil equilibrar las demandas de cambio de los usuarios y las quejas simultáneas en contra de tales cambios porque creaban problemas de retro-compatibilidad y, en consecuencia, trabajo adicional para las bibliotecas.

Mirando atrás en la revisión de las tablas de la CDU desde 1993, queda claro que las recomendaciones del informe del Grupo de trabajo fueron respetadas, especialmente en lo que respecta a la transformación de las clases revisadas de formato enumerativo a facetado. La aplicación del análisis de facetas tuvo un efecto inmediato en la notación de la CDU, la cual es mucho más expresiva. De esta forma, siempre que fue posible, los conceptos simples se representaron con notación simple y compuesta, y los conceptos complejos se construyeron mediante combinación. En muchos casos, el "facetado" significó una reestructuración completa de clases, lo cual condujo a que la clase entera fuera cancelada y reemplazada por una nueva tabla facetada. Cuando no se disponía de otra posibilidad, los propios números eran reutilizados para expresar nuevos significados. Esta es una de las consecuencias menos convenientes que puede causar la revisión, y en la clase 2 (Religión), por ejemplo, tal efecto no pudo ser evitado. En esta clase, los viejos números tuvieron que ser reutilizados con un nuevo significado: así, por ejemplo, en el esquema antiguo 22 era usado para Biblia, y en el nuevo esquema, tal número es empleado para Religiones originadas en el Lejano Oriente, mientras que Biblia se expresa con el número 27-23.

Otra acción que tuvo fuerte impacto en la CDU fue la remoción de duplicaciones de conceptos en varias tablas. La repetición de conceptos fue reemplazada por la reutilización de las mismas notaciones básicas para construir expresiones complejas, a menudo usando combinaciones mediante dos puntos y obteniendo, en consecuencia, notaciones más largas pero, al mismo tiempo, más fáciles de buscar, descomponer y comprender. Por ejemplo, se pueden combinar 028.8 (Lectura. Sugerencias para lectura) y 615.861 (Psicoterapia) para expresar 028.8:615.861 (Biblioterapia). O bien puede combinarse 342.7 (Derechos humanos fundamentales) con 612.3 (Reproducción (biología humana)) para expresar 
342.7:612.6 (Derechos reproductivos). Además, la introducción, en 1999 y 2003, de auxiliares comunes de propiedad, relaciones y procesos significó que muchos conceptos generales y recurrentes podían ser eliminados de las tablas principales y representarse a través de la combinación con auxiliares comunes. El ejemplo que mejor ilustra estos importantes cambios estructurales es la revisión de la clase 9, introducida en 1994:

$\begin{array}{ll}914 & \text { Geografía de Europa } \\ 914.10 & \text { Geografía de las Is. Británicas } \\ 940 & \text { Historia de Europa } \\ 941 & \text { Historia de las Is. Británicas }\end{array}$

$\begin{array}{ll}\text { reemplazada por } & 91(4) \\ \text { reemplazada por } & 91(41) \\ \text { reemplazada por } & 94(4) \text { "..." } \\ \text { reemplazada por } & 94(41) \text { "..." }\end{array}$

Aunque la expresiva notación en la cual el número principal se distingue claramente del auxiliar común puede parecer, a primera vista, una complicación innecesaria, especialmente para el ordenamiento en los estantes, el cambio es lógico y constituye un avance desde el punto de vista de la indexación y la recuperación de la información con la CDU en un ambiente virtual. En ambos casos el número se construye desde 94, agregando los auxiliares comunes de lugar (4) y (41) respectivamente, pero en la CDU antigua esto se lograba a través del método conocido como división paralela. Con la división paralela, una vez que el número se construye, la notación se amalgama en un número simple: el vínculo entre la representación original y el significado se pierde, y los elementos constructivos originales no pueden recuperarse en el proceso de búsqueda. Si los componentes se yuxtaponen y permanecen diferenciados y claramente marcados por notaciones expresivas, el esquema efectúa importantes avances en tres aspectos:

- Consistencia: el mismo concepto tiene siempre la misma representación en la notación; en la búsqueda, seremos capaces de encontrar todos los documentos relacionados con un mismo lugar o tiempo a través de diferentes campos del conocimiento, por ejemplo, Arte, Historia, Geografía, Ciencias Sociales, etc.

- Flexibilidad: con elementos de notación que son expresivos podemos cambiar y alterar el orden de citación de los elementos en la forma que más convenga a nuestras necesidades; así, podremos elegir entre 94"19"(44) o 94(44)"19".

- Accesibilidad verbal mejorada: pueden asignarse (en forma controlada) expresiones verbales a conceptos simples representados con notación simple, es decir, pueden usarse descriptores en la búsqueda.

En resumen, el pensamiento subyacente al trabajo de revisión ha sido influenciado por varios factores desde 1990. La revisión radical de clases enteras y la reutilización de números de la CDU fueron justificadas por un plan para alcanzar la revisión completa del esquema en diez años, después de lo cual el sistema 
sería estable; podría ofrecerse entonces a los usuarios un sistema nuevo, enteramente compatible para reemplazar los antiguos números. El uso de notaciones expresivas más largas y/o de combinaciones mediante dos puntos para anotar conceptos compuestos se justificó por el hecho de que, en un ambiente virtual, la eficiencia de la búsqueda puede ser más importante que la longitud de la notación. La velocidad y el volumen de los cambios, y su control, han sido considerados menos problemáticos en el uso virtual de la CDU, especialmente porque las tablas se difunden y emplean en forma electrónica.

\section{Desarrollos actuales y pasos a futuro}

A comienzos del 2000 ya era claro que el período de diez años programado para una revisión general de la CDU no era realista, especialmente cuando se lo confrontaba con el tamaño y la complejidad del esquema y la falta de recursos disponibles. Dado que las condiciones y la complejidad de las tareas pendientes no habían cambiado, el trabajo de revisión de la CDU continuará al mismo ritmo más allá de 2007. Tras el retiro de I. C. McIlwaine en 2006, María Inés Cordeiro fue elegida como nueva editora en jefe, y se estableció un nuevo equipo editorial.

La política del nuevo equipo es continuar con la revisión y modernización de la CDU, siguiendo principios previamente establecidos: mejorar la capacidad de síntesis del esquema y reducir la enumeración de materias complejas expresadas por notación simple. Este objetivo puede lograrse siguiendo la lógica del análisis de facetas y asegurando que, siempre que sea posible, un concepto general siempre es representado por la misma notación a lo largo del esquema.

Además de continuar con el trabajo de revisión editorial, otros varios aspectos necesitaron reorganizarse. Se trata principalmente de asuntos relacionados con las necesidades de los actuales usuarios en términos de alinear la gestión y los productos de la CDU al mundo virtual, y el modo en que los vocabularios controlados se comparten, acceden e implementan dentro de sistemas de información.

Estudios realizados sobre las áreas de aplicación de la CDU han revelado que el entorno web y los catálogos bibliotecarios ofrecen muchos casos que justifican el uso de la clasificación para la exploración del conocimiento y la expansión de las búsquedas (Slavic, 2005, 2006, 2006a). Sin embargo, esto depende en gran medida de búsquedas verbales y de la legibilidad de los datos mediante computadoras. En particular, los datos de la clasificación necesitan ser expuestos de tal forma que el esfuerzo humano -tanto en la implementación como en el uso- se reduzca al mínimo. Esto supone el empleo de la CDU no sólo como un conjunto de datos "nominales", sino también como "inteligencia" detrás de la pantalla, implicando que ha sido apoyada por sistemas de gestión de vocabulario central (o, para usar terminología biblioteconómica, por sistemas de control de autori- 
dades). El primer requerimiento futuro que enfrenta el UDCC es proveer los medios necesarios para que los datos de la CDU puedan ser fácilmente incorporados por sistemas de control de vocabulario; en la práctica, se buscará generar exportaciones del MRF nuevas y diversificadas, de tal forma que resulte más fácil y económico de implementar y usar.

Otra área importante para tener en cuenta en futuros desarrollos es la comunidad de usuarios de la CDU. Investigaciones recientes mostraron que la CDU es usada en 125 países y que existen traducciones a 39 idiomas. La CDU es el principal sistema de clasificación en unos 30 países, donde se provee capacitación, publicación y apoyo en forma regular (Slavic, 2005, 2008). Sin embargo, hay una comunidad mayor, distribuida a lo largo de otros 90 países, dónde la CDU es raramente enseñada en las escuelas de biblioteconomía, y para los cuales no existen ediciones recientes en el idioma local. Mientras que en la década de los noventa el UDCC se enfocaba principalmente hacia un pequeño número de grandes usuarios, ahora es posible usar el entorno informativo global para alcanzar y apoyar a un mayor número de usuarios de la CDU a nivel mundial.

Tomando en cuenta este marco general de preocupaciones e ideas, los siguientes puntos fueron abordados por el nuevo equipo editorial.

\subsection{Ampliar el equipo editorial a un cuerpo de colaboradores internacionales, y facilitar la comunicación con los usuarios}

Para sacar el mejor partido posible de tan valiosa entrada, los procesos de revisión deben ser cuidadosamente planeados y gestionados, para así evitar yuxtaposiciones innecesarias y derroche de tiempo, y para proveer un apoyo adecuado a los voluntarios. En 2007, la editora en jefe renovó la estructura organizacional del equipo editorial de la CDU, cuya forma actual puede representarse mediante dos círculos concéntricos. En el círculo interior se encuentra un grupo de seis especialistas en clasificación, que trabajan como editores asociados en estrecha cooperación con la editora en jefe, y que tienen la posibilidad de dedicar parte de su tiempo a tareas prácticas y técnicas (creación de planes y listas de tareas, control, corrección, y establecimiento, supervisión y coordinación de proyectos particulares de revisión), además de encontrar y cooperar con otros especialistas de materia. El círculo externo de colaboradores consiste en el UDC Advisory Board, actualmente compuesto por especialistas de materia de diferentes campos, procedentes de más de 20 países. Los miembros del Advisory Board pueden contribuir con opiniones expertas en problemas específicos, pero también a través del contacto y la inclusión de especialistas de materia de sus propios países.

Otro aspecto importante para la ampliación de la colaboración es la comunicación con los propios usuarios y la apertura de más oportunidades para el trabajo en red y las discusiones. Se planea concretar este aspecto a través de conferencias y talleres regulares de la CDU. 


\subsection{Renovar la infraestructura tecnológica y la gestión de los datos: una nueva base de datos y un sistema de apoyo editorial para la CDU, diversificación de los productos de la clasificación y mejora de sus datos}

Después de más de 13 años trabajando en forma única con CDS/ISIS, el UDCC ha considerado la necesidad de un nuevo sistema que permita gestionar el MRF y sus salidas mediante tecnologías avanzadas, especialmente mediante el uso de bases de datos relacionales sumadas a tecnologías web. Las versiones de prueba de la nueva base de datos ya están disponibles, y se espera que el nuevo sistema sea totalmente operativo para abril de 2009. Al respecto, la migración al nuevo sistema será acompañada de mejoras en los conjuntos de datos del MRF y de acciones de limpieza y corrección relacionadas con la consistencia de los datos. Tales acciones incluirían tanto la adición de nuevos campos como cambios en los ya existentes. Otro aspecto importante que también impulsó la renovación tecnológica del sistema de gestión de datos de la CDU tiene que ver con las salidas del MRF. Se necesita mejorar la provisión de formatos de exportación de dicho archivo, es decir, las formas de transferir los datos del MRF a los editores y usuarios. Esto será logrado haciendo que los datos de la UDC estén disponibles en formatos estándar como MARC 21, UNIMARC y SKOS, así como varios formatos de texto y exportación XML (Cordeiro y Riesthuis, 2006; Slavic, Cordeiro y Riesthuis, 2007).

\subsection{Actualizar y estructurar politicas, directrices, planes y procedimientos para los proyectos de revisión}

En 2009 se darán los primeros pasos para revisar, mejorar y consolidar la documentación sobre políticas, directrices y procedimientos de revisión. Este proceso es necesario no sólo porque siempre existen aspectos de política discutibles, sino también porque hay una necesidad real de detallar algunos de los criterios editoriales y, especialmente, de establecer procedimientos claros ahora que esperamos la contribución de un grupo de gente más amplio. Para facilitar el trabajo colaborativo internacional y sacarle el mayor partido posible al valioso tiempo de aquellos que contribuyan, las políticas y procedimientos de revisión deberán ser explicadas claramente, y se necesitarán instrucciones prácticas y detalladas para diferentes tipos de trabajo sobre el esquema.

\subsection{Potenciar las traducciones del esquema y la capacitación en su empleo}

La traducción de la CDU es una actividad costosa que consume mucho tiempo. En muchos países con escasos usuarios, los editores pueden no contar con incentivos suficientes para lanzar nuevas ediciones. Una forma de superar este 
problema y de ayudar a los usuarios es alentar el trabajo voluntario, proveyendo acceso al MRF de la CDU a aquellos que estén interesados en hacerse cargo de una traducción sin fines de lucro. Un buen ejemplo de tal idea es la edición sueca en línea, que posee alrededor de 6.000 números y que es generada en la escuela de biblioteconomía de Boras. Se basa exclusivamente en el trabajo voluntario y sin fines de lucro, principalmente como medio de capacitación.

\section{Conclusiones}

En el presente artículo hemos presentado un repaso general de la historia del desarrollo y la revisión de la CDU, pues este conocimiento es clave para comprender qué está ocurriendo y qué se pretende para el futuro del sistema de clasificación. Un compromiso importante del nuevo equipo editorial es ayudar a los usuarios a comprender qué ocurre con la CDU, cuáles son las ventajas y los beneficios de los cambios y cómo pueden ser mejor aprovechados.

El principal objetivo de la política de mantenimiento y desarrollo de la CDU en los años venideros es continuar el trabajo iniciado en 1993 para actualizar todo el sistema. Al hacer esto, el plan también es mejorar el control sobre los cambios y su consistencia, y asegurar que los usuarios reciben un apoyo completo durante el seguimiento y la implementación de tales cambios en su ambiente. Adicionalmente, se realizarán esfuerzos para proveer al sistema con un índice alfabético y vínculos con otros sistemas de materias, de forma que se aumente su poder de indexación y recuperación. La idea principal tras las exportaciones estandarizadas y los nuevos datos enriquecidos es disminuir los costos de producción e implementación de la CDU, así como aumentar el valor monetario de todos sus productos.

\section{Bibliografia}

Broughton, V. (1998): "The revision process in UDC: an examination of the systematic auxiliary of 'Point of View' using facet-analytical methods". En Extensions and Corrections to the UDC, n.20, pp. 17-20.

Cordeiro, M. I., y Riesthuis, G. (2006): "A new editorial support system for UDC". En Extensions and Corrections to the UDC, n. ${ }^{\circ} 28$, pp. 17-22.

Dahlberg, I. (1971): “Possibilities for a new Universal Decimal Classification”. En Journal of Documentation, 27 (1), pp. 18-36.

FID Internal Documentation (1976): UDC reform work. C 75-35. La Haya.

FID Internal Documentation (1990): Task Force for System Development: final report. La Haya.

FID Internal Documentation (1991): Guidelines for the creation of a standard version of UDC. [UDC-CONS 91-54, UDC Ref. 91-5]. La Haya.

Foskett, A.C. (1973): The Universal Decimal Classification: the history, present status and future prospects of a large general classification scheme. Londres: Clive Bingley. 
Freeman, R. R., y Atherton, P. (1969): "Final report of the research project for the evaluation of the UDC as the indexing language for a mechanized retrieval system". En Proceedings of the First Seminar on UDC in a Mechanized Retrieval System conducted by R. R. Freeman and P. Atherton, Copenhagen, 2nd-6th September, 1968. Copenhague: Danish Centre for Documentation, 1969 (FID/CR Report no 9), pp. 29-37.

Gilchrist, A. (1992): “UDC: the 1990s and beyond». En Williamson, N.J. y Hudon, M. (eds.). Classification research for knowledge representation and organization: proceedings of the 5th International Study Conference on Classification Research, Toronto, Canada, 24-28 June 1991. Amsterdam: Elsevier Science Publishers; La Haya: FID, pp. 69-78.

Lloyd, G. A. (1972): «UDC: revise or relegate? FID's Standard Reference Code project and UDC improvement programme». En Aslib Proceedings, 24 (10), pp. 580-587.

McIlwaine, I. C. (1990): "The work of the System Development Task Force». En Gilchrist, A. y Strachan, D. (eds). The UDC: essays for a new decade. Londres: Aslib, pp. 19-28.

McIlwaine, I. C. (1993): «UDC: the present state and future development". En 59th IFLA Council and Conference, Barcelona, Spain 22-28 August 1993, booklet 4, pp. 37-39.

McIlwaine, I. C. (1995): “UDC centenary: the present state and future prospects». En Knowledge Organization, 22 (2), pp. 64-69.

McIlwaine, I. C. (1996): «New wine in old bottles: problems of maintaining classification schemes". En Green, R. (ed.). Knowledge organization and change: proceedings of the Fourth International ISKO Conference, 15-18 July 1996, Washington DC. Frankfurt/ Main: Indeks Verlag, pp. 122-136.

Slavic, A. (2005): "The use of classification in the networked environment: the case of UDC». PhD thesis. University of London, University College London, 2005. [Disponible en la University of London Library y en la University College London Library].

Slavic, A. (2006): "The level of use of Universal Decimal Classification in library OPACs: pilot study 2004-2005" [En línea] disponible en <http://dlist.sir.arizona.edu/1688> [Consulta: 10 de enero de 2008].

Slavic, A. (2006a): "UDC in subject gateways: experiment or opportunity?». En Knowledge Organization, 33 (2), pp. 67-85 [En línea] disponible en <http://dlist.sir.arizona. edu/1556> [Consulta: 10 de enero de 2008].

Slavic, A. (2008): "Use of the Universal Decimal Classification: a worldwide survey". En Journal of Documentation, 64 (2), pp. 211-228. Pre-print disponible en <http://dlist. sir.arizona.edu/1555> [Consulta: 10 de enero de 2008].

Slavic, A.; Cordeiro, M. I., y Riesthuis, G. (2007): «Enhancement of UDC data for use and sharing in a networked environment". En Librarian Workshop in conjunction with The 31st Annual Conference of the German Classification Society on Data Analysis, Machine Learning, and Applications, March 7-9, 2007, Freiburg i. Br., Germany [En línea] disponible en <http://dlist.sir.arizona.edu/2093> [Consulta: 10 de enero de 2008].

Wellisch, H. (1971): "Reorganization of the UDC». En Nachrichten für Dokumentation, 22 (2), pp. 55-63.

Williamson, N. J. (1990): “The UDC: its future». En Gilchrist, A. y Strachan, D. (eds.). The UDC: essays for a new decade. Londres: Aslib, pp. 29-32.

Williamson, N. J. (1994): "The future revision of the UDC". En Extensions \& Corrections to the UDC, n.16, pp. 19-27. 